\title{
Predicting perfect pores
}

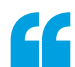

\section{Although the phenomenon has been theoretically studied, its contribution to hydrogen storage capa- city of porous materials has not been recognized}

Storing hydrogen safely and with sufficient storage capacity is needed to use hydrogen as a fuel in vehicles and other onboard applications. Typically hydrogen is stored under high pressures but large amounts of energy are required for this process, making it too expensive as well as impractical for use in vehicles. Porous materials - such as metalorganic frameworks (MOFs) - are one family of materials that can store gases under low pressure and, hence, it is key to identify ideal MOFs that are well-suited to these applications.

Now, writing in Advanced Materials, Jian Zhang, Wei Zhou, Banglin Chen and colleagues have devised two empirical equations that

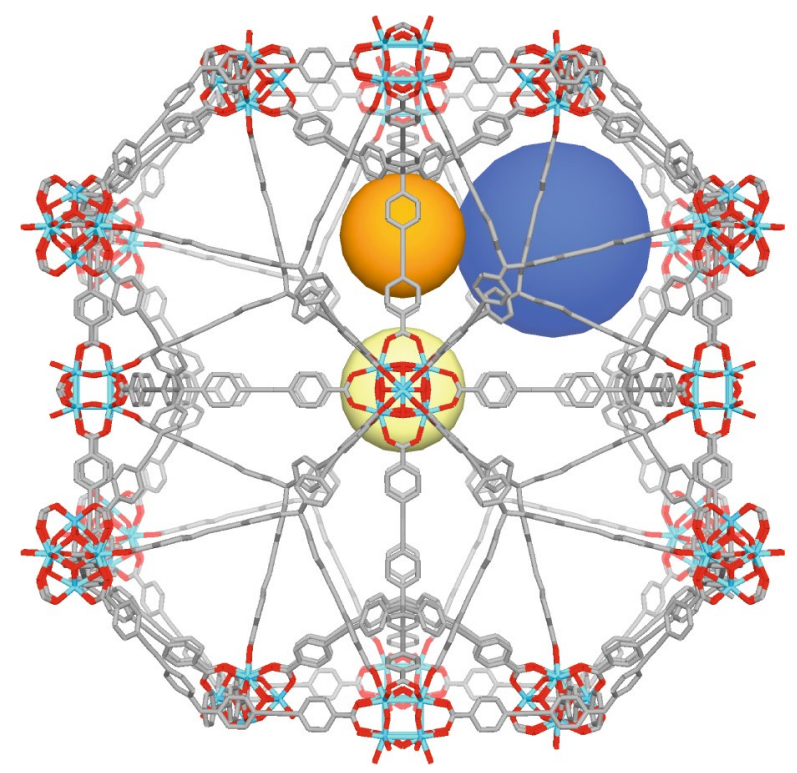

Credit: Reprinted with permission from Zhang, X. et al. (2020), Wiley-VCH predict the hydrogen storage capacity of MOFs with different pore volumes. Their findings aid our understanding about how pore properties influence the adsorption capacity of MOFs. "These equations demonstrate superior prediction accuracy to established equations indicating the contribution of pore occupancy and pore geometry is indeed significant," says Chen.

From this information, the researchers design and synthesize a zirconium-based MOF, namely NPF-200 (NPF, Nebraska Porous Framework) (see image), with a high absorption capacity for hydrogen and a record high volumetric working capacity $\left(37.2 \mathrm{~g} \mathrm{~L}^{-1}\right)$ between pressures of 100 and $5 \mathrm{bar}$, and at a temperature of $77 \mathrm{~K}$.

"More specifically, we show that pore occupancy decreases as pore volume increases owing to weaker sorbate-sorbent interaction in larger pores," says Xin Zhang, first author of the study. "Although the phenomenon has been theoretically studied, its contribution to hydrogen storage capacity of porous materials has not been recognized." In addition, pore occupancy is governed by the geometry of the pores, with those pores that are cage-like having a higher capacity to adsorb hydrogen than channel-like pores of the same pore volume.

Using the equations, the optimal pore volume to achieve a high capacity of hydrogen storage is identified as $3.3 \mathrm{~cm}^{3} \mathrm{~g}^{-1}$. However, for practical applications, it is also necessary for the porous material to have a high volumetric adsorption capacity, which arises from a high volumetric surface area. With this information in hand, the highly porous material, NPF-200, was identified with the potential to adsorb a high volume of hydrogen owing to its pore volume of $2.17 \mathrm{~cm}^{3} \mathrm{~g}^{-1}$ and volumetric surface area of $2,268 \mathrm{~m}^{2} \mathrm{~cm}^{-3}$.

"The encouraging results of NPF-200 guided by the empirical equations in this study motivate us to explore new MOFs with optimal pore volume and geometry to realize even higher hydrogen storage capacity," says Chen. "With the convenient structural modelling and theoretical prediction of pore properties, more MOF materials are expected to be discovered with higher storage capacities soon," concludes Chen. Also, it is likely that these equations could be applied to other families of highly porous materials, for example, activated carbon, zeolites, covalent organic frameworks and hydrogen-bonded organic frameworks, and hence identify the best materials for hydrogen storage from these families.

Alison Stoddart

ORIGINAL ARTICLE Zhang, X. et al. Optimization of the pore structures of MOFs for record high hydrogen volumetric working capacity. Adv. Mater. https://doi.org/10.1002/adma.201907995 (2020) 